\title{
Maternal deaths from bleeding associated with caesarean delivery: A national emergency
}

In this issue of the SAMJ, Fawcus et al. ${ }^{[1]}$ draw attention to the epidemic of avoidable maternal deaths from caesarean-related haemorrhage in South Africa (SA). How can this epidemic be addressed?

Firstly, the most effective and cost-effective way to reduce maternal mortality from all causes is by preventing unintended pregnancies. In SA we have proved our capacity to mainstream the largest HIV treatment programme in the world into all levels of our health services. We need to do the same for family planning. In particular, the copper intrauterine contraceptive device (IUCD) needs to be made more accessible as an option. Current limited evidence suggests that the IUCD is more effective than injectable progestogens. ${ }^{[2,3]}$ Health workers need training in counselling and techniques of inserting the IUCD after pregnancy termination, after vaginal birth and at caesarean section, as well as in interval insertion.

Secondly, we need to reduce unnecessary caesarean sections (CSs) by improving the quality of childbirth care. Drivers of the increasing CS rates include erosion of obstetric skills and confidence, and spiralling litigation, which leads to 'defensive', unscientific practices and reduces the funds available for public health services. Companionship during childbirth ${ }^{[4]}$ has been shown to reduce CSs. In metropolitan areas, primary care, midwife-led childbirth services can be improved in terms of uptake, quality, safety and costeffectiveness by location on the premises of large hospitals. ${ }^{[5]}$

Thirdly, we need to ensure that women undergoing CS receive adequate prophylactic uterotonics. Ironically, the availability of intravenous access at CS has led to a preference for intravenous rather than intramuscular oxytocin administration, but because of the risk of hypotension specific to the intravenous route, the initial dosage has been reduced from 10 to $2.5 \mathrm{IU}$, supplemented by an ongoing infusion that may or may not be remembered. ${ }^{[6]} \mathrm{A}$ case may be made for the simplicity and certainty of the intramuscular route. In addition, given the increased risk and danger of haemorrhage in the context of CS, addition of ergometrine for women without contraindications, and a second dose of oxytocin after 4 hours, have been recommended.

As the authors point out, ${ }^{[1]}$ the popularity of misoprostol has tended to distract attention from the more effective conventional uterotonics. Its formulation as a simple, inexpensive tablet may be assumed to be a less 'invasive' and therefore safer option than injectable uterotonics. On the contrary, misoprostol is a powerful prostaglandin analogue with multiple side-effects. It has a modest effect on blood loss after childbirth, but has not yet been shown to reduce the overall risk of death. ${ }^{[7]}$

What about future research? Oxytocin has the limitation of requiring a cold chain to maintain effectiveness. The World Health Organization is currently testing the effectiveness of a new, heatstable formulation of carbetocin. ${ }^{[8]}$ More evidence is needed on the relative effectiveness of alternative strategies to treat haemorrhage when it occurs. No doubt our knowledge will increase. But to address the current crisis of avoidable maternal deaths, what is needed most are the health service infrastructure, education, workforce and political will to make the care already known to be effective, accessible to all women.

\section{G J Hofmeyr}

Walter Sisulu University, Mthatha, Eastern Cape, South Africa, University of Fort Hare, Alice, Eastern Cape, University of the Witwatersrand, Johannesburg, South Africa, and Eastern Cape Department of Health

\section{Corresponding author: G J Hofmeyr (justhof@gmail.com)}

1. Fawcus S, Pattinson RC, Moodley J, et al., for the National Committee on Confidential Enquiries into Maternal Deaths (NCCEMD). Maternal deaths from bleeding associated with caesarean delivery: A national emergency. S Afr Med J 2016;106(5):472-476. DOI:10.7196/SAMJ.2016.v106i5.1082

2. Hofmeyr GJ, Singata M, Lawrie TA. Copper containing intra-uterine devices versus depo progestogens for contraception. Cochrane Database Syst Rev 2010; Issue 6. Art. No.: CD007043. DOI:10.1002/14651858.CD007043.pub2

3. Hofmeyr GJ, Singata-Madliki S, Lawrie TA, Bergel E, Temmerman M. Effects of the copper intrauterine device versus injectable progestin contraception on pregnancy rates and method discontinuation among women attending termination of pregnancy services in South Africa: A pragmatic randomise controlled trial. Reprod Health (in press).

4. Hodnett ED, Gates S, Hofmeyr GJ, Sakala C. Continuous support for women during childbirth. Cochrane Database Syst Rev 2012, Issue 10. Art. No.: CD003766. DOI:10.1002/14651858.CD003766. pub4

5. Hofmeyr G, Mancotywa T, Silwana-Kwadjo N, Mgudlwa B, Lawrie TA, Gülmezoglu A. Audit of a new model of birth care for women with low risk pregnancies in South Africa: The primary care onsite midwife-led birth unit (OMBU). BMC Pregnancy Childbirth 2014;14(1):417. DOI:10.1186/s12884014-0417-8

6. Farina Z, Fawcus S. Management of obstetric haemorrhage. Oxytocin ensuring appropriate use and balancing efficacy with safety. S Afr Med J 2015;105(4):271-274. DOI:10.7196/SAMJ.9179

7. Hofmeyr GJ, Gülmezoglu AM, Novikova N, Lawrie TA. Postpartum misoprostol for preventin maternal mortality and morbidity. Cochrane Database Syst Rev 2011, Issue 2. Art. No.: CD008982. DOI:10.1002/14651858.CD008982

8. Widmer M, Piaggio G, Abdel-Aleem H, et al. Room temperature stable carbetocin for the prevention of postpartum haemorrhage during the third stage of labour in women delivering vaginally: Study protocol for a randomized controlled trial. Trials 2016;17(1):143-152. DOI:10.1186/s13063-016-127-1

S Afr Med J 2016;106(5):435. DOI:10.7196/SAMJ.2016.v106i5.10822 